Liu Jingwen ${ }^{1}$

DOI 10.31902/LL.2021.8.1b

\title{
LA CHINE DE JUDITH GAUTIER : UNE ETUDE DU CHAPITRE “LA VALLEE DU DAIM BLANC" DU DRAGON IMPERIAL
}

Résumé : Après la publication de la traduction des poèmes traditionnels chinois sous le titre Le Livre de Jade, Judith Gautier fait connaître au lecteur français son talent dans le domaine romanesque. Son premier roman Le Dragon impérial au sujet chinois est différent des œuvres précédentes grâce à son originalité dans plusieurs aspects, comme la description détaillée du paysage taoïste, ses réflexions profondes sur la philosophie taoïste. De plus, elle inclut ces réflexions dans la construction et le déploiement de l'intrigue. Cette recherche fondée sur la lecture attentive du chapitre "La Vallée du daim blanc" du Dragon impérial, vise à révéler l'originalité de sa représentation littéraire de la Chine.

Mots clés : l'orientalisme, les cultures comparées, le taoïsme, l'image de la Chine.

\section{Introduction}

Judith Gautier (1845-1917) se fait connaître en 1867 aux lecteurs français grâce à la publication (sous le pseudonyme de Judith Walter) de sa traduction de poèmes traditionnels chinois, Le Livre de jade. Elle étend son activité littéraire, un peu plus tard, au domaine romanesque en composant Le Dragon impérial. Le dernier publié en 1869 est le seul roman au sujet chinois de Judith Gautier.

La passion des lettrés et des érudits français pour la Chine peut remonter au dix-septième siècle. Des œuvres à sujet chinois sont accessibles au public à partir de l'époque-là: L'Orphelin de la Chine de Voltaire, Contes chinois (1827) et Iukiao-li, ou les deux cousines (1826) traduits par Abel-Rémusat et Théâtre chinois ou choix de pièces de théâtre (1838) traduits par Antoine Bazin, etc. Ce qui distingue Le Dragon impérial des œuvres précédentes qui se réfèrent à la Chine peut être que le récit se déploie avec pour arrière-plan des réflexions et des interprétations philosophiques. Elle considère le tao comme "culte de la raison" (Judith Gautier Les Peuples étrangers 97). Par ailleurs, son intérêt pour la philosophie taoïste s'étend plus loin

\footnotetext{
${ }^{1} \mathrm{PhD}$ candidate at Sorbonne Université, France.
} 
dans Les Peuples étrangers dans lequel elle consacre un chapitre à part entière à l'étude la médecine chinoise fondée sur le principe du yin et du yang. Dans Le Dragon impérial, ces réflexions sont souvent dévoilées soit en vers libre, soit à travers les descriptions détaillées des éléments chinois, de sorte qu'elles suscitent de la curiosité pour la culture chinoise chez le lecteur français. À propos du Dragon impérial, Victor Hugo écrivit dans une lettre à Judith Gautier datée du 4 août 1872:

J'ai lu votre Dragon impérial. Quel art puissant et gracieux que le vôtre ! Cette poésie de l'extrême Orient, vous en avez l'âme en vous, et vous en mettez le souffle dans vos livres. Aller en Chine, c'est presque aller dans la lune. Vous nous faites faire ce voyage sidéral. On vous suit avec extase et vous fuyez dans le bleu profond du rêve, ailée et étoilée. (1952: 213)

Mallarmé écrivit : "Merci du Dragon impérial, doublement cher : une grande merveille, n'est-ce pas?" (1959: 307). Dans cette "grande merveille", Yvan Daniel fit remarquer comme suivant :

Les péripéties du roman d'aventures autant que les justifications "historique", et politique de l'intrigue laissent ainsi toujours apparaître un intérêt curieux pour la pensée chinoise, bien qu'elle soit encore mal ou très imparfaitement comprise. Si Judith Gautier ne s'intéresse pas au bouddhisme dans ce roman, elle fait allusion au confucianisme et au taoïsme [...] (40)

En conservant sa propre âme rêveuse teintée de son imagination, Judith Gautier était tentée de créer "une grande merveille", c'est-à-dire qu'une fois que Judith Gautier a décidé de faire de la Chine un miroir magique par lequel elle voit un monde de croisements, un mélange d'idéal et d'imagination pour l'Europe, la connaissance réelle reculait à l'arrière-plan. Judith Gautier, en tant qu'orientaliste attirée par une civilisation plus ancienne, invitait son lecteur au voyage en Chine, un pays reconstruit et manipulé dans son texte fermé. La représentation de la Chine dans Le Dragon impérial est établie autour de "la préférence exotique [qui] se double presque toujours d'une attirance pour certains contenus au détriment d'autres" (Todorov, 1989: 299). Tandis qu'elle apprécie tant de valeurs en Chine: le minimalisme dans la vie sociale et économique, la conformité à la nature et la philosophie taoïste, il convient de 
noter qu'elle néglige le réalisme et le matérialisme des Chinois, le despotisme et les institutions hiérarchiques de l'empire. Néanmoins, tout est représenté d'une manière sélective et agrandissante. Le Dragon impérial ne permet pas au lecteur de profiter d'une vue panoramique sur la Chine. Chaque aspect de la Chine y inclus est, pour une bonne part, témoigne de l'intention qu'a Judith Gautier de créer l'altérité culturelle, sociale et philosophique. Le minimalisme dans la vie sociale et économique prôné par la philosophie chinoise arme mentalement l'écrivaine contre la crise morale, l'accumulation de richesses et la vanité répandues à son époque; le paysage taoïste offre à Judith Gautier une nouvelle vision sur le rapport que l'homme engage avec le tout; plus de réflexions sur les doctrines taoïstes dans "La Vallée du daim blanc" visent à la fois à approfondir sa compréhension du rapport entre l'homme, la volonté et le monde et à déployer une histoire logique selon le principe "préparation et paiement".

\section{L'Orientalisme de Judith Gautier}

Il convient de noter qu'au dix-neuvième siècle, les véritables voyages en Chine sont encore réservés à quelques missionnaires, ambassadeurs et militaires. Même si Judith Gautier n'a jamais mis les pieds en Chine, sa passion pour la culture chinoise, en dépit de ses connaissances indirectes, lui permet d'éprouver un choc culturel, un bouleversement des valeurs de la tradition européenne. La mode de la chinoiserie s'est développée en Europe entre le dix-huitième et le dix-neuvième siècle grâce à l'importation des soieries, porcelaines, et d'autres produits qui stimulaient l'intérêt pour la Chine chez les artistes.

Judith Gautier fut initiée à la connaissance de la Chine en aidant son père à ranger les documents qu'il lisait à propos de ce pays lointain. Ce pays mystérieux et son parfum exotique intriguent l'esprit curieux de Judith Gautier. Il est raisonnable d'attribuer sa passion pour la Chine à l'influence déterminante de son père, Théophile Gautier. Les œuvres de ce dernier inspiré par la Chine, tels que le roman Le Pavillon sur l'eau et le poème intitulé "Chinoiserie", représentent le monde extrême-oriental sous la forme d'un mystère attractif. Les connaissances que Judith Gautier a pu acquérir sur la Chine ne sont pas le seul fruit de l'influence de son père. Nous évoquons ici en particulier Tin Tun Ling, homme de lettres chinois. Il est devenu un familier des Gautier après s'être réfugié en France et était communément 
appelé "le Chinois de Gautier1". Théophile Gautier l'a hébergé chez lui et l'a embauché à partir de 1863 pour qu'il enseigne le chinois et la littérature chinoise à sa fille Judith, laquelle a été immédiatement fascinée par la langue et la poésie chinoises. Il est probable qu'une grande partie des connaissances sur la Chine de cette jeune femme française provient de cet homme de lettres chinois qui avait vécu le bouleversement social et la rébellion politique en personne avant de venir en France. L'influence de ce professeur chinois explique en grande partie pourquoi Judith Gautier peut fournir une description détaillée et vraisemblable de la Chine du dix-septième et du dix-huitième siècles incluant l'instabilité politique et religieuse dans Le Dragon impérial.

À part Théophile Gautier et Tin Tun Ling, il ne faudrait pas négliger l'autre célèbre sinologue d'alors, Stanislas Julien. Titulaire de la chaire de langue et littérature chinoises au Collège de France de 1832 à 1873, Stanislas Julien est réputé pour sa passion pour la langue et la poésie chinoises, et ses ouvrages traduits du chinois, par exemple, Meng Tseu (1828), Tchaochikou-eul ou l'orphelin de la Chine_(1834), Le livre de la voie et de la vertu (1842), Notices sur les pays et les peuples étrangers, tirées des géographes et des historiens chinois (1846), etc. De plus, le cercle de Stanislas Julien et celui de Judith Gautier s'entrecroisent dans la vie. Lors d'un entretien en 1910, où elle fut élue à l'Académie Goncourt, Judith Gautier mentionna la venue de Stanislas Julien chez elle. Malgré les différentes méthodes employées par Judith Gautier et Stanislas Julien dans leurs études de la culture chinoise, il n'y a aucune raison pour que Judith Gautier néglige la contribution de Stanislas Julien à la sinologie à leur époque.

Remy de Gourmont a remarqué: “À s'en tenir à ses romans, à ses poésies, à ses pièces de théâtre, Judith Gautier serait plus volontiers chinoise que française" (1919: 136). Judith Gautier, "princesse chinoise incarnée"2, incarne dans la littérature française contemporaine le goût de l'Orientalisme spécifiquement tourné vers la Chine. L'Orientalisme, selon Edward Saïd, est "en fin de compte une vision politique de la réalité, sa structure accentue la différence entre ce qui est familier (l'Europe, l'Occident, "nous») et ce qui est étranger (l'Orient, "eux»)" (2005: 59). 
Il n'y a aucun doute, en effet, que la géographie et l'histoire imaginaires aident l'esprit à rendre plus intense son sentiment intime de lui-même en dramatisant la distance et la différence entre ce qui est proche et ce qui est très éloigné. (2005: 71)

Sur le plan géographique, la Chine est située plus de huit mille kilomètres à l'est de la France. Des textes largement imaginaires consacrés à la Chine, fondés sur des sources incertaines et indéterminées, dirigent la vision de l'Europe vers les divergences entre la Chine et l'Occident. L'opacité, l'isolement et le retard de la Chine, contrastés avec les échanges multilatéraux et l'accélération des pays européens, engendrent la perte du pouvoir de sa parole. Pour définir elle-même par contraste, l'Europe construit, ainsi, une opposition sinoeuropéenne en prenant la Chine pour son rival et antipode dans tous les domaines.

Apparenté à l'orientalisme, l'exotisme est aussi un relativisme qui existe dans les comparaisons entre l'Europe et "les autres". "Dans les deux cas, ce qu'on valorise n'est pas un contenu stable, mais un pays et une culture définis exclusivement par leur rapport avec l'observateur" (Todorov, 1989: 297). Depuis le dix-septième siècle, même si les Européens, surtout les Français, se passionnent pour les voyages à l'étranger, l'Europe maintient toujours une grande distance avec la Chine. Cette distance nécessaire met Judith Gautier en position intermédiaire entre connaissance et méconnaissance, de sorte qu'elle idéalise ce pays éloigné.

Pour renforcer la couleur exotique, la distance entre la Chine et l'Europe est mise en avant par Judith Gautier. Étant consciente que le progrès scientifique et la prospérité économique apportent la corruption européenne, Judith Gautier se tourne vers la Chine antique pour donner forme à son utopie extrême-orientale. Bien que l'arrière-plan historique du récit dans son roman soit tel qu'un homme se révolte contre Kangxi, qui fut l'empereur de la Chine de 1661 à 1722, il ne semble pas à Judith Gautier que les valeurs culturelles chinoises se renouvellent depuis la genèse du taoïsme. Ce qu'elle apprécie consiste en Chine antique. La Chine qui l'intéresse n'est pas seulement éloignée sur le plan géographique, mais aussi sur le plan temporel. Confinée dans son ancienneté, la Chine se fige et devient, de ce fait, l'inverse de l'Europe. 


\section{La Chine traditionnelle: le minimalisme}

L'Europe se situe dans le dynamisme de demande de biens de consommation au dix-neuvième siècle. Dans "Études morales sur la société française au XIX siècle. - la vraie cause de la crise", Emile Montégut a parlé de la crise de la société française provenant en partie "de l'accumulation des richesses dans certaines mains, de l'exploitation, de la mauvaise distribution des produits" (1851: 202). "Le mal c'est leur vanité, leur envie" (1851: 202), poursuivit-il.

Théophile Gautier a aussi critiqué son propre pays en écrivant dans Les Jeunes-France: "je partage l'avis des Orientaux: il faut être chien ou Français pour courir les rues quand on peut rester assis bien à son aise chez soi" (1878: XI). Bien que ces Orientaux dont Théophile Gautier a parlé soient les peuples du Proche-Orient et du Moyen-Orient, nous nous rendons compte qu'un esprit critique à l'égard de la société moderne européenne prépare et favorise son admiration pour l'Autre. Probablement influencée par Théophile Gautier, à la fois son père et mentor, Judith Gautier était également mécontente de ce qu'elle voyait autour d'elle. Cela a été témoigné par Anatole France:

"La pensée magnifique de madame Judith Gautier [...] ne vînt jamais dans nos climats humides et gris, qui ne sont point faits pour sa beauté rare [...] Quand le poète du Dragon impérial [...] quitte le monde féerique de l'Orient qu'elle a rêvé, de son Orient [...], quand elle entre dans les réalités de la vie moderne, elle perd dans nos brouillards sa grâce divine. (1923: 138)

Peu à l'aise dans la société européenne, Judith Gautier va loin jusqu'à l'inverse du goût de luxe et du désir possessif des Européens dans Le Dragon impérial. Avant d'aborder les dialogues à propos des réflexions taoïstes, Judith Gautier décrit la tenue élimée d'un philosophe chinois de manière détaillée:

Ce sage, ce philosophe, ce disciple de Kon-Fou-Tse et de Lao-Tse, portait une ample et longue robe déchiquetée et sale, de coton jaunâtre, aux larges manches plus longues que les bras, et serrée à la taille par une corde noire. Il avait la tête et les pieds nus. Il s'appuyait sur un long rameau tortueux. (1869: 232)

Héritant la sagesse du taoïsme, ce disciple se présente sous une apparence extérieure de frugalité dans le texte de Judith Gautier. Car la connaissance sur le peuple chinois des Européens 
du dix-neuvième siècle provient largement des premiers classiques chinois traduits dans lesquels la sagesse et l'âme noble côtoient de près le minimalisme sur la vie matérielle. La richesse matérielle n'est pas prônée ni par le confucianisme, ni par le taoïsme. "Les grandes richesses produisent les grands soins et les grands inquiétudes" (Confucius \& Foucher, 1783: 114). Dans Le tá hio, ou la grande étude: le premier des quatre livres de philosophie morale et politique de la Chine, ce point de vue est répété: "les richesses ornent et embellissent une maison, la vertu orne et embellit la personne; dans cet état de félicité pure, l'âme s'agrandit [...] C'est pourquoi le sage doit rendre ses intentions pures et sincères" (Confucius, 2012: 53). Jacques Sancery fait remarquer les rapports entre la vie spirituelle et la vie matérielle dans la traduction comme suivant:

Indifférent au luxe, fuyant la vie fastueuse des grands, contre les excès [...] Le Maître étonne et déconcerte plus d'un par la simplicité de ses désirs et son absence de besoin: "Une nourriture frugale, de l'eau à boire, la tête qui repose sur un bras replié: voilà le bonheur! Honneurs et richesses mal acquis ne sont pour moi que nuages qui passent!" (2009: 205)

De plus, Étienne Badimont note aussi que "le confucianisme était aussi à l'origine d'une mystique de l'éducation orientée vers l'action, d'une conduite économique inspirée par la frugalité" (1996: 203). De même, le taoïsme déclare que le Maître embrasse la simplicité et que le sage taoïste pratique aussi la vertu de la frugalité de la vie. Cette dernière devient un art de vivre sans austérité, simplement, détaché des contraintes matérielles.

Qu'est-ce qui nous est le plus précieux, de notre personne ou de nos richesses?

Quel est le plus grand malheur, de les acquérir ou de les perdre?

[...]

Celui qui sait se suffire est à l'abri du déshonneur.

Celui qui sait s'arrêter ne périclite jamais.

Il pourra subsister longtemps. (Lao Tseu, 1842: 67)

Julien Stanislas ajoute des notes à propos de ce chapitre:

Ce que les hommes cupides recherchent avec ardeur, ce sont les richesses, et pour les acquérir, ils vont jusqu'à exposer leur vie; ils ignorent que leur personne est plus précieuse que les richesses [...] 
Les richesses l'enflent d'orgueil; pour les obtenir, il accable le peuple de fatigues, il appauvrit le royaume, il trouble ses esprits, il expose son cœur à une foule de désirs, il se met en révolte contre le Tao [...] l'univers le déteste, le monde lui déclare la guerre [...] (1842: 217)

Ce point est répété par Lao Tseu dans le chapitre XLVI:

Il n'y a pas de plus grand crime que de se livrer à ses désirs.

Il n'y a pas de plus grand malheur que de ne pas savoir se suffire.

Il n'y a pas de plus grande calamité que le désir d'acquérir.

Celui qui sait se suffire est toujours content de son sort. (1842: 69)

On ne s'étonnera donc pas de trouver l'image du sage chinois pratiquant sa philosophie du minimalisme matériel dans Le Dragon impérial. La simplicité de vie matérielle promue par les philosophes chinois est mise en avant dans le roman pour mettre en évidence l'écart entre culture chinoise et culture européenne. Car cette simplicité s'oppose à l'expansion du désir dans la société française où elle vit. Judith Gautier ne décrit pas seulement la tenue frugale, mais aussi l'environnement d'habitation du sage chinois:

L'empereur suivit le philosophe et entra dans la caverne. Il jeta les yeux autour de lui. L'habitation du Solitaire était d'une simplicité complète: un amas de feuilles sèches formait le lit, deux rochers étaient le siège et la table; pour tout ustensile, une écuelle de porcelaine ébréchée [...] (1869: 233)

Les mœurs taoïste et la vie innocente proche de la nature sont représentées comme les antipodes du monde occidental dans le roman. Les Chinois conçoivent leur vie sous les auspices de la simplicité, et non du luxe, du gaspillage ou de la consommation excessive. Le sage chinois se libère des contraintes matérielles, se nourrit dans la nature pour cultiver son esprit et obtient, enfin, la tranquillité intérieure et la sagesse. Par l'expérience solitaire d'une vie de simplicité et d'indépendance, en cultivant son esprit, le "Solitaire grave et serein" (1989: 232) sous la plume de Judith Gautier parvient à trouver sa dignité d'être humain sans se couper de ses racines naturelles, ni de sa place naturelle dans l'univers. 


\section{Le paysage Shanshui: une autre perception de la nature}

Le paysage conquérait une place importante en Europe au dixneuvième siècle. Cet engouement pour le paysage pénétrait dans l'art et la littérature. Les lettrés et artistes se sont assortis d'un intérêt pour le paysage dans leurs créations littéraires, car "la nature y est aussi belle que l'art, dont la mission est de la spiritualiser: là, le paysage a des idées et fait penser" (Balzac, 1843: 2). Ces idées, faisant l'objet de diverses sociétés et expressions esthétiques, sont étroitement liées à la perception du paysage, autrement dit, à un point de vue. Ainsi, la découverte du paysage lointain devient non seulement celle d'un patrimoine naturel qui donne une identité géographique, mais aussi une rencontre culturelle avec une vision et des valeurs nouvelles.

La perception de la nature par les Chinois pourrait être une autre facette pour expliquer la préférence de Judith Gautier pour la culture chinoise. Le chapitre XXI du Dragon impérial commence par une longue description du paysage naturel dans le style de Shanshui ${ }^{3}$ :

Sur les pentes des montagnes humides et brillantes se répandaient d'onduleuses cascades [...] Les sommets qui déchirent les nuages paraissaient fumer lentement, et au fond de la vallée, le lac qui les reflète était de cristal bleu.

Sur les plateaux des Montagnes Fleuries [...] Les amandiers en fleur, les pechers, les abricotiers sauvages, le mûrier et les figuiers rampants s'enlacent [...] un grand oiseau aux pattes grêles traverse l'eau et jette un cri. (1869: 225, 226)

La description de la nature sous la plume de Judith Gautier est empreinte de cosmologie taoïste d'où dérive l'art de Shanshui. Cette nature se fait connaître par l'assemblage des montagnes, des nuages, des fleurs et arbres, des eaux et des animaux. Judith Gautier laisse parler la nature comme un artiste en fournissant des scènes purement naturelles loin de la société. Dans la description de Judith Gautier, "l'allure libre et aisée d'une nature qui ne manifeste aucune volonté et ne laisse voir aucun travail dans la production de ses effet" (Rancière, 2020: 31) se montre à travers l'intrication des lignes onduleuses et des contours indéfinis. Ce sont la variété des choses, comme des végétations diverses, les nuages et l'oiseau, et les inégalités du terrain, comme les pentes et les plateaux des montagnes, le lac et le fond de la vallée, qui animent le paysage dans le texte de Judith 
Gautier. Pourtant, cette multiplicité d'éléments se fond en un effet de Shanshui, un effet d'ensemble qui lie le tout aux parties. "Par définition, la peinture de montagnes et eaux (Shanshui) est incompatible avec la ville (Chengshi ou Dushi): elle ne dépeint jamais de paysage urbain" (Escande, 2005: 16). Le terme "paysage" est passé du japonais au chinois vers la fin du dixneuvième siècle. Depuis lors, Shanshui et "paysage" ne se distinguent pas l'un de l'autre.

Il serait juste de dire que l'idée de Shanshui et la perception de la nature par les Chinois ne sont rien d'autre que l'inversion et l'antipode de ce que signifie le mot "paysage" en français. Le dernier désigne "étendue de terre couverte par le regard". Premièrement, le suffixe "-age" fait lier l'idée de paysage à l'action d'arranger ou d'assembler les objets pour présenter l'ensemble du pays. Deuxièmement, il s'agit du "regard de l'homme", c'est-à-dire que le mot "paysage" a pour objet d'exprimer ce que l'homme perçoit à travers son regard. Finalement, ce "territoire" désigne "région ou état" qui inclut non seulement les lieux sauvages et naturels, mais aussi les lieux civilisés à l'intérieur des villes. Nous dirions que le paysage en français n'est pas une existence indépendante de l'homme. Il devient un objet observé, arrangé et contrôlé par l'homme. Sur le plan de la peinture, les Européens présentent le paysage en utilisant la perspective, à travers laquelle l'espace est organisé et ordonné sous la vision humaine. Les Européens établissent une distance entre la nature et l'homme en pensant que les lieux naturels éloignés de la civilisation humaine sont souvent considérés comme sauvages. Le mot "sauvage" dérive du latin silva (forêt). Il semble que la liberté naturelle non subie de l'influence de la part de l'homme devient une raison d'être inférieure au monde humain.

Bien différente de l'art du bel arrangement des produits de la nature en Europe, la description du paysage chinois dans le texte de Judith Gautier met en lumière une nature dont "la rudesse [des] éléments et les irrégularités de leur disposition offrent à qui sait les regarder les principes de la juste unité du divers en un tout: celle qui se fonde sur le contraste" (Rancière, 2020: 44). Cette idée de contraste fait écho au principe de l'art de Shanshui. Le paysage dans le roman de Judith Gautier se montre à travers une absence de sélection. En Chine, le terme de Shanshui signifie littéralement "Montagnes-Eaux". L'idée de Shanshui veut 
dire les scènes naturelles comprenant les montagnes et les eaux. C'est le mot Shanshui qui servait à décrire le "paysage" en chinois. Sur le plan de la perception de la nature des Chinois, la montagne et l'eau sont les deux pôles inverses et contrastés mais compatibles, dans lesquels les animaux, les roches, les plantes et les humains trouvent leur source de vie. Judith Gautier nous offre une nature, toujours irrégulière et libre sans intervention de l'homme, qui n'est définissable que par elle-même dans sa spontanéité. La nature "ne distingue pas, elle laisse coexister toutes sortes d'objets, toutes variétés de formes, de couleurs, de lumière et d'ombre" (Rancière, 2020: 46). Le paysage dans le texte de Judith Gautier imite la manière dont la nature travaille aveuglément et l'attitude spirituelle qui dépasse toute maîtrise. Ce point, contrasté aux règles d'art du bel arrangement, correspond, en revanche, à la vertu de l'esthétique traditionnelle chinoise. On peut interpréter l'idée de création qui dirige l'art de Shanshui par le terme de ziran : ce qui se réalise de soi-même. Le caractère de la nature se fonde sur la fusion d'une infinité d'éléments non sélectionnés, sur son allure aisée et sur son esprit libéral. Ceux-ci naissent de l'âme universel de la nature qui entre en communion avec les artistes chinois. Le paysage, selon Michel Collot, est un lieu dans lequel une identité culturelle peut s'incarner et se construire. Comme le fait remarquer Collot, "le paysage n'est pas le pays, mais une image du pays"(2011: 80). De ce fait, l'âme nationale chinoise se montre sous la description du paysage de Judith Gautier. Le paysage décrit par elle dans le style de Shanshui, fortement teinté d'esthétique et de mœurs chinoises, joue un rôle important dans l'échange entre l'expérience sensible et la construction symbolique, entre identité culturelle chinoise et la tradition philosophique occidentale.

À part cela, une autre fonction du paysage dans son texte est d'ouvrir une voie de communication égale et réciproque entre nature et homme. Inspirée par l'art de Shanshui, Judith Gautier a créé une scène naturelle de la manière chinoise:

Les Montagnes Fleuries sont d'ordinaire désertes, et la Vallée du Daim Blanc est une vallée de solitude. Les jours sont rares où un pieux voyageur, venant du Hou-Pé ou du Ho-Nan, monté sur un buffle qu'il dirige du bout d'un rameau symbolique, suit le sentier à demi effacé qui s'enroule autour du mont et descend dans la vallée jalouse. 
Aucun bruit humain ne se mêle au chaud bourdonnement, épars dans la lumière, qui vient des arbres, des cascades, des fleurs, des papillons. (1869: 226, 227)

Dans cet environnement, les éléments ne sont pas arrangés selon la géométrie ou d'autres formes artificielles. Les montagne désertes, le sentier à demi effacé, le voyageur, le buffle et la vallée de solitude composent un univers uni qui devient "la métaphore d'un ordre naturel non perturbé" (Rancière, 2020: 49). Dans le texte de Judith Gautier, le "voyageur", autrement dit, l'homme, n'est plus différent de son "buffle", "des arbres, des cascades, des fleurs", etc. C'est-à-dire que le "voyageur" n'observe pas le paysage alentour, mais vit dans le paysage. L'homme qui ne joue plus le rôle de l'observateur et les composants non-humains de la nature qui ne sont plus observés par l'homme occupent une place égale dans l'environnement naturel. Tous les éléments, y compris l'homme, dans la scène, sont liés l'un à l'autre par une sympathie générale. D'une manière similaire à l'art de Shanshui, Judith Gautier, en créant des scènes originales de la nature, fournit au lecteur non seulement une peinture chinoise où il peut trouver le plaisir pittoresque mais aussi une ambiance qui lui permet de vivre une tranquillité et une retraite utopique comme un Chinois, ou plus précisément, comme un véritable ermite taoïste.

Le paysage dans le texte de Judith Gautier fait appel non seulement à une réforme esthétique mais aussi à une réforme de pensée. À mes yeux, l'intérêt essentiel pour la représentation du paysage chinois de Judith Gautier n'est pas seulement une mode d'ajouter la couleur exotique d'une région lointaine, mais une préparation qui correspond à un éclairage sur la mutation des mentalités occidentales. Dans Le Dragon impérial, la nature n'étant plus un objet observé par l'homme devient un relais actif entre les vertus des choses de la nature et les vertus de l'homme. À côté de l'ajout de la couleur exotique et de l'empreinte de taoïsme, la description de la nature chinoise dans le roman nous passe à réfléchir d'une manière chinoise. Purifiant, le paysage naturel permet à l'homme d'intérioriser le monde extérieur. Cette fois-ci, la nature n'est plus un objet passif arrangé ou manipulé par l'homme, au contraire, elle devient une existence active et dynamique qui exerce son influence sur l'homme. Quand l'empereur Kangxi se plonge dans la nature, Judith Gautier met en lumière la fonction soignante de la nature: 
Il se trouva bientôt seul et s'assit près d'un ruisseau, le sourire au lèvre, l'âme bienveillante; il ne songeait plus à son empire ni à sa gloire; il se sentait libre et enveloppé par la nature. (1869: 229)

Plutôt que d'être symbole des émotions ou des réflexions spirituelles de l'homme, "Les Montagnes Fleuries" et la végétation dans le roman deviennent eux-mêmes la spiritualité.

Le terme "nature" en chinois se compose de deux mots: zì rán. Zì rán est utilisé pour désigner la spontanéité avant la dynastie Qin (221 - 206 av. J.-C). L'expression Zì rán servait à l'origine à décrire "ce qui advient de soi-même" et a été étendu au "naturel" ou "nature" car aux yeux des Chinois, la nature, non soumise à des règles érigées par la société humaine. En Chine, dans la nature ou un état naturel il y a la loi universelle d'où découlent les choses justes et raisonnables. Dans le roman de Judith Gautier, la montagne, l'eau, les animaux sauvages, la forêt, tous ceux qui sont considéré comme sauvage en Europe constituent le point de départ et de fin des réflexions des Chinois. La Chine et son paysage sont présentés sous la plume de Judith Gautier en tant que l'image de l'Autre. La représentation littéraire du paysage chinois sous la plume de Judith Gautier dans son roman apporte un souffle nouveau sur l'évolution de la civilisation occidentale en fournissant au lecteur une nouvelle perception du paysage qui l'amène à "s'affranchir du dualisme invétéré de la pensée occidentale, à dépasser un certain nombre d'oppositions qui la structurent, comme celles du sens et du sensible, du visible et de l'invisible, du sujet et de l'objet [...] de l'esprit et du corps, de la nature et de la culture" (Collot, 2011: 18). Selon la tradition philosophique européenne, ces termes s'opposent ou se subordonnent l'un à l'autre. L'une des fonctions du paysage dans le texte de Judith Gautier est de nous inviter à goûter une autre expérience paysagère et d'instaurer ainsi "une interaction [...] qui nous invite à penser autrement" (Collot, 2011:18). L'exotisme de Judith Gautier ne se limite pas à la description du paysage pittoresque; il s'étend en revanche aux réflexions sur des doctrines taoïstes.

\section{La philosophie taoïste et la construction de l'histoire}

Il convient de noter qu'au long de l'histoire de la philosophie, la relation entre homme et Dieu préoccupe presque toujours les philosophes occidentaux. La philosophie occidentale révèle une 
compétition entre la religion et la raison. Alors que les philosophes du Moyen Âge mettaient Dieu au centre de leurs réflexions, l'humanisme était au cœur des préoccupations des philosophes de la Renaissance. Pendant le siècle des Lumières, le triomphe de l'homme et de la raison se manifestait dans les divers champs des sciences. Au dix-neuvième siècle, la société européenne mettait l'accent sur l'indépendance personnelle et la liberté de conscience en demandant à la religion les vérités morales et religieuses qui sont le fondement nécessaire de l'ordre social.

Pourtant, il n'existe pas en Chine antique d'expression équivalente du concept occidental de religion. Le terme zongjiao un néologisme provenant du japonais, n'est adopté que vers le début du vingtième siècle pour traduire le concept occidental de "religion". En Chine antique, avant l'influence du bouddhisme les Chinois n'adhèrent pas à une seule communauté religieuse mais pratiquent diverses doctrines philosophiques. La première différence entre la Chine et l'Europe s'avère évidente dans $L e$ Dragon impérial: il n'y a pas de Dieu en Chine. Judith Gautier écrit que "le ciel n'a pas de parents, il traite également tous les hommes. (1869: 233)" Ici, le rôle de Dieu est remplacé par le Ciel qui désigne la dimension des Principes suprêmes. Au lieu de s'adonner à la recherche du vrai maître du monde, les Chinois poursuivent l'harmonie entre l'homme et l'univers. La deuxième différence entre la Chine et l'Occident dans le roman est qu'en Chine, l'homme qui est considéré comme un objet, plutôt que de sujet n'est pas au cœur de l'univers. Dans le doctrine suivant - "le sage fait le bien comme il respire; c'est sa vie (Judith Gautier, 1869: 233)"- le taoïsme nie le droit d'intervention humaine et la place centrale et privilégiée de l'homme. Le fonctionnement du monde sous le Ciel ne permet pas l'intervention humaine dans le processus spontané des phénomènes. Non soumises à la force de l'homme, toutes les choses paraissent, se développent, disparaissent selon leurs propres lois. D'ailleurs, la respiration du sage dément, à la fois, la nécessité et l'état naturel à travers lesquels le bien est achevé comme il va de soi. Le but d'achever le bien exige la non-intervention qui n'est pas une attitude d'inaction mais une conformité active à l'ordre cosmique originaire. Par contraste avec le point de vue sur la place centrale et l'initiative de l'homme prévalant en Europe, Judith Gautier insère son interprétation du principe philosophique chinois - "le 
Tao pratique constamment le non-agir et (pourtant) il n'y a rien qu'il ne se fasse" (Lao Tseu, 1842: 53) - dans la communication entre le sage et le gouverneur de l'état. La troisième différence entre la Chine et l'Europe que Judith Gautier met en lumière dans son roman est l'égalitarisme universel du taoïsme. "Le ciel n'a pas de parents " (1869: 233) implique que le Ciel, différent de Dieu, n'est pas personnel. Vu que Judith Gautier prive l'homme de la place centrale et privilégiée, il serait plus juste d'interpréter sa phrase citée ci-dessus: le Ciel traite également tous les êtres, y compris les hommes. Le Ciel ici n'est plus le ciel que l'on voit dans la nature physique, mais l'incarnation de la loi universelle à laquelle tous les êtres se soumettent, comme Lao Tseu dit dans son Tao te king:

Ciel terre est sans bienveillance

Les dix mille êtres il les traite en chiens-de-paille

Le saint est sans bienveillance

Les cent familles il les traite en chiens-de-paille. (1842: 35)

L'impartialité de l'univers se lie souvent à l'égalitarisme qui est l'un des motifs que les Occidentaux attachent habituellement à l'image utopique de l'Autre. Au dix-huitième siècle, l'exotisme de Lahontan se manifestait à travers les Hurons qui "ne connaissent point la subordination militaire non plus que la civile" (1706: 145). Il affirme que "la nature ne connaît point de distinction, ni de prééminence dans la fabrique des individus d'une même espèce, aussi sommes-nous tous égaux" (1706: 257). Différente de l'image du "bon sauvage" (Todorov, 1989: 304) de Lahontan, le principe égalitariste du taoïsme présenté sous la manipulation sélective de Judith Gautier concerne non seulement la relation égalitaire des hommes mais aussi la relation égalitaire entre l'homme et les autres êtres. L'égalitarisme du taoïsme interprété par Judith Gautier en deux dimensions n'est rien que l'inversion de l'idéologie européenne. Sachant que, en réalité, les empires chinois sont fondés sur le système hiérarchique strict et sur la suprématie du pouvoir impérial et que la plupart des officiers chinois croient en le confucianisme plutôt que de taoïsme, la culture chinoise présentée dans Le Dragon impérial n'est qu'une manifestation de l'utopisme égalitariste de Judith Gautier.

Il convient également de noter qu'en Occident, les réflexions philosophiques depuis le cinquième siècle avant J.-C. 
reposent sur la dichotomie. Platon oppose la forme à la matière, et plus tard, Descartes le sujet à l'objet. En occident, la poursuite de la vérité se fonde ainsi sur la logique de l'analyse comme dichotomie et partition qui permettent de diviser un tout en deux parties. Néanmoins, il n'y a pas la dichotomie équivalente en Chine même si toutes les choses sont divisées en deux catégories opposées: le Yin et le Yang. Le Yin et le Yang sont opposés mais complémentaires et interchangeables. Il n'y a pas de frontière tranchante entre le Yin et le Yang car ils ne s'excluent pas, en revanche, le Yin peut se transformer en Yang et inversement. Dans Le Dragon impérial, le sage évoque à l'empereur le fait que "Qui trouve du plaisir dans le vice et de la peine dans la vertu est encore novice dans l'un et dans l'autre (1869: 233)". La leçon éthique contient aussi une vérité cosmique dialectique. On peut interpréter la doctrine comme suivant : la personne qui trouve du plaisir dans le vice, plongée dans la joie et le profit éphémères et immérités ignore toujours le danger potentiel; en même temps, la personne qui trouve la peine dans la vertu lors qu'il se sacrifie et délaisse son propre intérêt au profit de quelqu'un d'autre ne voit pas le bonheur et la fortune latents que sa vertu l'apportera. Selon le principe de la transmutation de Yin-Yang, le bonheur ne peut pas exister sans son contraire et ne cesse de tendre à se transformer vers l'autre bout. La relation entre "ceci" et "cela" dans le taoïsme n'est pas le même que le rapport entre "soi" et "l'autre" dans la philosophie occidentale. Le principe de l'alternance de "ceci" et "cela" est considéré comme la condition de toute existence.

Le malheur

Marche au bras du bonheur

Le bonheur

Couche au pied du malheur (Lao Tseu, 1842: 165)

La couleur chinoise est renforcée dans le roman non seulement par le paysage pittoresque mais aussi par des réflexions taoïstes qui sont mises dans la construction de l'histoire: l'émeute se cache sous la tranquillité trompeuse de la ville; le danger s'approche quand l'empereur est content de la paix superficielle; le sage propose à l'empereur une robe "informe, grise, fétide", car l'apparat auguste manifestant son statut supérieur ne peut qu'apporter le danger en route pour rentrer à la capitale, il n'est plus meilleur qu'un vieux vêtement. 
Le bien et le mal se transforment l'un vers l'autre. Sur le plan de la réflexion philosophique et la logique à l'égard du déploiement de l'intrigue, la Chine mise en dehors de la logique européenne met en cause la dichotomie entre le bien et le mal dans le roman. De cette manière, la Chine est définie encore une fois comme l'autre de l'Occident.

À côté de l'objectif de créer un pays lointain à l'antipode de l'Occident, la mise en place de la méditation et des éléments (le paysage et le daim blanc) taoïstes dans "La Vallée du daim blanc" consiste, par ailleurs, à rendre l'histoire cohérente dans un cadre logique. Juste après un round de chasse, le paysage est décrit comme un élément inspirant :

[Les collines] méritent bien leur nom de Montagnes Fleuries, car ici le sol est un parterre brillant, le vent un parfum, le son une musique. Qu'il serait doux de vivre en ces lieux, exempt de soucis et d'attachement, car Lao-Tse a dit: La perfection consiste à être sans passions pour mieux contempler l'harmonie de l'univers [...] il ne songeait plus à son empire ni à sa gloire; il se sentait libre et enveloppé par la nature [...] (Gautier, 1869: 229)

La description n'est pas sans importance, car elle sert de signe a priori préparant le terrain pour l'introduction des doctrines philosophiques chinoises lors de la rencontre de l'empereur avec le sage.

Le daim blanc apparaît sur la scène presque simultanément mais il ne suscite plus l'ambition du chasseur de le tuer. Au contraire, l'empereur le protège et sauve de la menace d'un ours. Le cerf blanc, selon la religion taoïste, symbolisant la prospérité et l'immortalité est souvent présenté comme la monture de Shoulao, le dieu taoïste de la longévité. On retrouve souvent le cerf blanc dans les peintures classiques chinoises se tenant à côté des sages taoïstes. La méditation taoïste de Kang-Si dans la nature et sa rencontre avec un animal fétiche dans le terrain de chasse servent d'une préparation pour la suite si l'on inclure les dans la construction et l'évolution de l'intrigue. Elles peuvent être également considérées comme un mini-nœud dramatique qui bouleverser la trajectoire et l'ambiance violente de chasse. La trajectoire de chasse de Kang-Si dans la vallée commence par la confrontation entre le loup et les cavaliers, la lutte terrible entre le loup et l'épervier, en passant par méditation taoïste et la rencontre avec le daim blanc jusqu'au sauvetage et à 
la rencontre avec le sage. La violence de l'empereur est bouleversée par des réflexions spirituelles (sa méditation dans la nature) et la rencontre avec le daim blanc. Ces dernières ont leurs utilités. Dans ce processus, le désir de tuer chez Kang-Si baisse. La tuerie de l'ours n'est pas pour le posséder, mais pour sauver le daim. La forme de possession du daim devenant plus tard de l'amener avec lui, plutôt que de le détruire, implique une diminution de l'obstination à tuer. Dans ce chapitre, le daim conduit Kang-Si à le poursuivre jusqu'à rencontre le sage solitaire. À travers la distance entre l'empereur et le daim, parfois proche, parfois loin, contrôlé par le daim, on se rend compte que le dernier semble avoir l'intention d'amener Kang-Si chez le sage. Placé après le paysage qui éveille la médiation de l'empereur et une série de comportements intentionnels du daim mythique qui sert traditionnellement de compagnon ou monture des dieux taoïstes, le moment où Kang-Si rencontre le Solitaire et lit les maximes gravées sur des rochers est bien préfiguré et peut être considéré comme un paiement de la préparation. Il est désormais très logique pour le lecteur que la rencontre et la conversation entre l'empereur et le sage se produisent suit au guide, soit spirituel, soit symbolique, teinté de couleur taoïste, bien que la préparation apparaisse sous les formes d'existence que le protagoniste ne sait pas quand il servira.

Le cercle "préparation et paiement" ne se clôture pas par ce moment-là. L'introduction des maximes gravées sur des rochers sert, à la fois, le paiement de la préparation précédente étudiée ci-dessus et la préparation elle-même pour les prochains paiements. La dernière est manifestée dans la destinée des deux personnages principaux Ta-Kiang et Yo-Mei-Li.

Ta-Kiang, un laboureur, s'obstine dans l'idée de changer son destin et de conquérir le pays. Il répète le désir et la volonté de saisir le trône, par exemple, dans le chapitre XIX: "Ta-Kiang, durant trois lunes, a crié : 'Je suis le Frère Aîné du Ciel ; je libère et je glorifie ! [...] Je reprendrai le nom de la lumineuse dynastie et je m'assiérai sur un trône rouge et fumant [...]" (Gautier, 1869: 206) et plus tard, dans le chapitre XXVII: "Bientôt je me reposerai sur mon trône" (Gautier, 1869: 278). À propos de Yo-Mei-Li, une jeune fille s'obstinant à aimer Ta-Kiang qui ne l'aime jamais. Elle insiste bien que Ta-Kiang exprime effectivement son refus:

Mais Ta-Kiang dit avec rudesse: 
- Je n'ai pas besoin qu'une femme me suive:

- Une femme! s'écria Yo-Mei-Li en résistant aux larmes qui lui montaient aux yeux. J'au revêtu les habits de mon jeune frère et j'ai pris un cœur d'homme en même temps que ce costume d'homme. S'il faut du courage pour te servir, j'en aurai plus qu'un guerrier ; s'il faut de l'adresse et de la ruse, je serai plus adroite qu'un voleur et plus rusée qu'un juge ; s'il faut mourir, je mourrai, et morte, s'il faut revenir des pays d'en haut pour te servir encore, sois tranquille, j'en reviendrai. (Gautier, 1869: 15)

La destinée tragique de Ta-Kiang et Yo-Mei-Li est préfiguré par les doctrines taoïstes - "le ciel n'a pas de parents, il traite également tous les hommes", "le sage fait le bien comme il respire; c'est sa vie (Gautier, 1869: 233)". L'évolution de l'histoire ne suit pas la volonté de l'homme, car la loi suprême de l'univers est impartiale sans bienveillance. Le bien est achevé non par l'intervention imposée et piloté par la volonté, mais par l'accord avec les lois harmonieuses de la nature sans perturber les choses. De plus, dans le chapitre "La Vallée du daim blanc", un autre avertissement taoïste mis en parallèle avec d'autres maximes est "accueillez vos pensées comme des hôtes, et traitez vos désirs comme des enfants " (Gautier, 1869: 233). On peut l'interpréter ainsi: il est important de mettre à l'aise vos hôtes et de donner assez de liberté à vos enfants; traitez vos pensées et désirs comme vous traitez tes hôtes et enfants, il ne faut donc pas tenter d'imposer la volonté obstinée ou obsédante sur les pensées et désirs. Cet avertissement fait écho au principe de le non-agir (wuwei) qui est illustré dans Tao Te King: "Il pratique le non-agir, et alors il n'y a rien qui ne soit bien gouverné (Lao Tseu, 1842: 12)". Selon Lao Tseu, tous outrepassant ce principe sont voués à l'échec. En incluant ces pensées philosophiques, Judith Gautier prépare le dénouement dans lequel Ta-Kiang subit l'échec de la rébellion et Yo-Mei-Li est abandonnée lors que Ta-Kiang refuse de l'épargner comme otage au prix d'abandon d'attaque militaire. Car plus les deux personnages s'obstinent à leurs désirs, plus capable le lecteur est de prévoir leur fin tragique.

\section{Conclusion}

Si on peut dire que les représentations stéréotypées de l'exotique sont souvent liées à l'image du sauvage depuis le dix-sixième siècle, Judith Gautier se débarrasse de cette limite. Ce que Judith Gautier présente dans son texte est un autre monde civilisé qui n'était pas marqué par l'humanisme et la philosophie 
occidentaux. Différent de Jean-Jacques Rousseau qui met l'accent sur le culte du primitivisme et la société sauvage, Judith Gautier incarne l'image de la Chine par le biais de l'art de Shanshui, par la philosophie et les mœurs chinoises. En premier lieu, loin du monde sauvage, ce monde extrême-oriental dispose de toutes formes de la civilisation, parmi lesquelles l'art chinois devrait être "d'un apport durable à la civilisation universelle" (Lin, 2003: 375). Le paysage chinois dans le texte de Judith Gautier devient un miroir qui reflète l'âme nationale des Chinois se cachant sous une apparence physique peu remarquable. Dans Les Beaux-arts en Europe de Théophile Gautier, les Chinois ont cheveux d'un noir bleu, les yeux oblique et bridés, les sourcils d'encre de Chine, les ongles longs et transparents. En bref, Théophile Gautier donne un portrait des chinois comme suivant: "les hommes arrondissent leurs larges ventres et étagent leurs triples mentons, tandis que les femmes sont minces sous leur tunique de soie"(1878: 135). Pourtant, À travers le paysage dans le style de Shanshui, les mœurs et pensées philosophiques chinoises, Le Dragon impérial, en évitant les représentations stéréotypées de l'exotique, révèle une âme harmonieuse en communion avec la nature sous les visages rudes, une émotivité profonde derrière les apparences sérieuses et solennelles, et une sagesse idéaliste sous leurs doigts fins et jaunes.

Néanmoins, il convient de noter qu'il faut toujours place la naissance d'une œuvre littéraire au cœur d'un contexte culturel et historique. Et Le Dragon impérial n'est pas une exception. En prenant en compte des données culturels et historiques, nous nous rendons compte que l'image de l'Empire du Milieu se trouve dans la nécessité de s'établir comme l'antipode du monde occidental. Car, selon Bernard Franco qui cite le point de vue d'Edward Saïd et en tire la relativité de l'Orient, "il n'existe pas d'Orient absolu, l'Orient est toujours l'orient d'un occident [...]" (2016: 308). L'image de la Chine est ainsi un système de représentation. Dans ce roman, la Chine est restituée et représentée par sa porte-parole française qui fait agrandir la couleur et les caractéristiques chinoises, de sorte que la dernière porte l'empreinte du pouvoir de l'auteure et de l'Occident et ne soit plus ainsi une réalité absolue.

Par conséquent, la Chine est transformée en volonté de l'auteure elle-même et, en même temps, en reflet de l'autorité collective européenne de l'époque de Judith Gautier. De cette 
manière, Judith Gautier représente un monde lointain "moitié imaginé, moitié connu" où il y a "des monstres, des démons, des héros; des terreurs, des plaisirs, des désirs" (Saïd, 2005: 80). Il s'agit moins de la description d'un peuple réel que de la formulation d'un idéal dans ce roman. La représentation de la Chine doit être conforme aux résultats des études académiques, aux recherches anthropologiques et biologiques, aux expositions dans les musées: un peuple, ayant le culte de la nature et nourri par la civilisation taoïste. Fondée sur une conscience occidentale hégémonique, la représentation de la nation chinoise perdant l'aspect réel devient une image vue à travers le prisme de l'Occident. La partie active, sociale, et réaliste dans le caractère des Chinois est effacée, en revanche, le "non-agir" et ni "autre" ni "soi" du taoïsme et la conformité à la Nature sont mis en évidence dans Le Dragon impérial. Par admiration pour le taoïsme qui fonde l'image idéalisée des Chinois dans son roman, Judith Gautier aborde d'abord la description de la scène naturelle imprégnée de style de Shanshui, et ensuite, présente une scène de dialogue philosophique entre le sage et l'empereur Kang xi en pleine nature. Le dialogue a lieu entre un ermite éloigné de la société et un gouverneur de la société. Le dernier a le pouvoir suprême, alors que le premier lui révèle le "non-agir". Leur communication, qui est une rencontre des opposés sans incompatibilité ou conflit, dénote, en revanche, une grande souplesse et tolérance. Judith Gautier prend la sagesse taoïste pour les principes. Ce qu'elle cherche à la Chine, c'est tout ce qui y est différent de l'Europe; ce qu'elle veut faire par le biais de l'écriture est de retrouver une Chine taoïste, qui a plus de chances de ressembler à ses rêves.

L'image de la Chine se forme dans le roman de Judith Gautier par la mise de l'accent sur les traits de la Chine différents de l'Europe et la négligence de ce qu'elle partage en commun avec l'Europe. Aux yeux de Judith Gautier, le taoïsme est sans aucun doute préférable au confucianisme pour fabriquer une Chine dont l'idéal spirituel est regardé comme l'esprit furtif et vagabond. En effet, le confucianisme mettant en valeur le rôle social de l'homme et les institutions sociales, semble partager trop de choses en commun avec la philosophie européenne pour qu'il favorise l'édification d'un Autre orientalisé. Apparenté à l'humanisme européen qui met l'homme au centre de l'univers, l'humanisme confucéen implique que sa priorité est aussi 
l'épanouissement humain et social. Le positivisme et l'humanisme du confucianisme pourrait réduire l'étrangeté des Chinois et la distance entre la Chine et l'Europe. Cela pourrait expliquer le choix, par lequel Judith Gautier se sert du taoïsme, au lieu du confucianisme, pour formuler une Chine idéale. Le chapitre XXI du roman attache de l'importance à la Nature, physique et métaphysique, à un état sans intervention humaine. Pourtant, les principes du fonctionnement de la société humaine ne côtoient pas tout le temps ceux de la Nature. L'histoire que l'empereur voyager et méditer dans la nature dénonce implicitement l'esprit furtif et vagabond. Cela rejoint à l'image populaire des Chinois au dix-neuvième siècle en Europe au dixneuvième siècle: les Chinois sont dénués d'initiative et d'énergie.

La Chine devait exister inévitablement comme l'inverse et l'antipode de l'Occident. Ainsia, loin d'exister indépendamment, les Chinois, aux yeux des Européens, mènent une vie plus près de la nature et de l'enfance. Grâce à cette vie, les émotions et l'instinct se donnent libre cours, de sorte que la vie des Chinois devient un art et la philosophie un retour conscient à la simplicité, l'idéal le réel. Il y a une distance entre ce genre de construction idéale et la Chine réelle. Les Chinois ne sont pas pratiquants ou croyants d'une seule religion. Ils combinent le positivisme confucéen, le sens du réel, le mépris de la logique, et les doctrines taoïstes rêveurs. De cette façon, les Chinois peuvent avoir un caractère équilibré. Leur attitude envers le monde "rendra possibles une vraie jouissance de notre séjour terrestre et l'éclosion d'un tempérament plus pacifique, plus raisonnable, moins bouillant" (Lin, 2003: 39). Yutang Lin a écrit ainsi dans L'importance de vivre:

Elle [La philosophie chinoise] est plus grande que Confucius, plus grande que Laozi, elle les transcende, eux et les anciens philosophes; elle puise en eux ses pensées, les accorde en un tout, et, des contours abstraits de leur sagesse, elle a créé un art de vivre charnel, visible, palpable et compréhensible pour le commun des hommes. (2003: 39)

Néanmoins, dans Le Dragon impérial, les Chinois perdent leur tempérament équilibré. Ce fait est imputable en partie à la domination d'une culture sur une autre. Ils ne parlent pas pour eux-mêmes, c'est Judith Gautier qui parle pour eux et dit à ses lecteurs français ce que sont la Chine et son peuple. "Exotisme" est donc synonyme d'“altérité" (Todorov, 1989: 359). Afin 
d'obtenir une image contrastante de l'Autre, l'auteure sélecte partiellement ce qui l'intéresse, souvent ce qui est à l'inverse de l'Europe en négligeant la correspondance de ce qu'elle représente avec une Chine réelle.

\section{Notes}

1 “Celui qu'on a souvent appelé 'le Chinois de Théophile Gautier' vécut plusieurs années chez les Gautier" (Yvan Daniel, "Judith Gautier et la Chine", <https://heritage.bnfffr/france-chine/fr/judith-gauthierchine-article $>$ ).

${ }^{2}$ Judith Gautier se plaisait à dire d'elle-même: "Je suis chinoise. Je suis même la réincarnation d'une princesse chinoise" (Camacho).

${ }^{3}$ Shanshui signifie littéralement "montagne et eau".

\section{Références bibliographiques}

Badimont, Étienne. Socrate ou Confucius: essai sur le devenir de la Chine et de l'Occident. Paris: FeniXX, 1996.

Balzac, Honoré de. La Comédie humaine. Scène de la vie de province, in: Oeuvres complète. Vol. 5. Paris: Furne, 1843.

Camacho, Mathilde. Judith Gautier: sa vie et son œuvre. Paris: Droz, 1939.

Collot, Michel. La Pensée-paysage. Arles: Actes Sud, 2011.

Confucius. Entretiens. Trad. Séraphin Couvreur. Paris: Culture Commune, 2013.

---_Le Tá Hio, ou la grande étude: le premier des quatre livres de philosophie morale et politique de la Chine. Trad. Guillaume Pauthier. Paris:

L'Institut de France, 1837.

Confucius, \& Foucher, Simon._La Morale de Confucius: philosophe de la Chine. Trad. Jean de Labrune. Paris: Valade, 1783.

Daniel, Yvan. Littérature française et culture chinoise, 1846-2005. Paris: Indes savantes, 2011.

---."Judith Gautier et la Chine." Web. 22 June 2020

$<$ heritage.bnf.fr/france-chine/fr/judith-gauthier-chine-article>.

Escande, Yolaine. Montagnes et eaux, la culture du shanshui. Paris: Hermann, 2005.

Franco, Bernard. La Littérature comparée: histoire, domaines, méthodes. Malakoff: Armand Colin, 2016.

France, Anatole. La vie littéraire. Vol. 4. Paris: Calmann-Lévy, 1923.

Gautier, Judith. Le Dragon impérial. Paris: Alphonse Lemerre, 1869.

---.Le Livre de jade. Paris: Alphonse Lemerre, 1867.

---. Les Peuples étrangers. Paris: G. Charpentier. 1879.

Gautier, Théophile. Les Beaux-arts en Europe. Série I. Pairs: Michel Lévy Frères, 1855.

---. Les Jeunes-France. Paris: G. Charpentier, 1878. 
Gourmont, Rémy de. Promenades littéraires. Vol. 3. Paris: Mercure de France, 1919.

Hugo, Victor. CEuvres complètes de Victor Hugo. Vol. 3. Paris: Librairie Ollendorff, 1952.

Javary, Cyrille. Le Yi Jing: le grand livre du yin et du yang. Paris: Fides, 1989.

---. Les Trois Sagesses chinoises: taoïsme, confucianisme, bouddhisme. Paris: Albin Michel, 2012.

Lahontan, Louis Armand. Mémoires de l'Amérique septentrionale, tome II. La Haye: Charles Delo, 1706.

Lao Tseu. Lao Tseu Tao te king: le livre de la voie et de la vertu. Trad. Stanislas Julien. Paris: Imprimerie Royale, 1842.

Leiva, Antonio Momínguez and Muriel Détrie. Le Supplice oriental dans la littérature et les arts. Neuilly-lès-Dijon: Éditions du Murmure, 2005.

Lin, Yutang. La Chine et les Chinois. Paris: Éditions Payot \& Rivages, 2003.

---. L'Importance de vivre. Trad. J. Biadi. Arles: Édition Philippe Picquier, 2004.

Mallarmé, Stéphane. Correspondance, 1862-1871. Recueillie, classée et annotée par H. Mondor et J.P. Richard. Paris: Gallimard, 1959.

Montégut, Emile. “Études morales sur la société française au XIXe siècle. — la vraie cause de la crise actuelle." Revue des deux mondes. Tome 12 (1851): 201-222.

Rancière, Jacques. Le Temps du paysage: aux origine de la révolution esthétique. Paris: La Fabrique Éditions, 2020.

Saïd, Edward. L'Orientalisme. Trad. Catherine Malamoud. Paris: Édition du Seuil, 2005.

Sancery, Jacques. Confucius. Paris: Cerf, 2009.

Todorov, Tzvetan. Nous et les autres: la réflexion française sur la diversité humaine. Paris: Édition du Seuil, 1989. 